\title{
Synthesis of Silver Nanoparticles in Kaolinite and Their Antibacterial Behaviour
}

\author{
Salmah Moosa \\ Agrotechnology and Bioscience \\ Division, \\ Malaysian Nuclear Agency \\ Selangor, Malaysia
}

\author{
Anis Nadia Mohd Faisol Mahadeven, \\ Agrotechnology and Bioscience Division, \\ Malaysian Nuclear Agency Selangor, \\ Malaysia
}

\author{
Kamyar Shameli \\ Department of Environmental \\ Engineering and Green Technology, \\ MJIIT University of Technology \\ Malaysia, Kuala Lumpur, Malaysia.
}

\begin{abstract}
Silver/kaolinite nanoparticles (Ag/Kln NPs) were prepared via chemical reducing method using Sodium borohydride $\left(\mathrm{NaBH}_{4}\right)$ as the reducing agents. Kaolinite was suspended in aqueous $\mathrm{AgNO}_{3}$ solution and the adsorbed $\mathrm{Ag}^{+}$ ions were reduced on the surface of kaolinite with $\mathrm{NaBH}_{4}$. The effect of physicochemical parameters such as concentrations of silver salt on the size, shape and distribution of silver nanoparticles were studied. The silver nanoparticles prepared were characterized by ultraviolet-visible spectroscopy, powder $x$-ray diffraction, fourier transform infrared spectroscopy, transmission electron microscopy, and field emissions scanning electron microscopy couple with energy dispersive $x$-ray spectrometer analysis to determine the properties of silver nanoparticles. The antibacterial activity of Ag NPs in kaolinite was investigated against Gram positive bacteria which is, Enterococcus faecalis and Methicillin-resistant Staphylococcus aureus as well as Gram-negative bacteria which is, Escherichia coli and Proteus vulgaris by the well diffusion method using Mueller-Hinton agar at different sizes of Ag NPs. All of the synthesized Ag/KIn NPs were found to show antibacterial activity. They exhibit numerous advantages from medical applications point of view. This finding from this study can potentially be used in various areas, including pharmaceuticals, cosmetics, foods, and medical applications.
\end{abstract}

Keywords- Silver kaolinite; nanoparticles; sodium borohydride; chemical reduction; silver nitrate

\section{INTRODUCTION}

Novel applications of nanoparticles and nanomaterials are growing rapidly on various fronts due to their completely new or enhanced properties based on size, their distribution and morphology [1]. They are a link between bulk materials and atomic or molecular structures and are being explored in various biotechnological, pharmacological and pure technological uses. Among these nanomaterials, silver nanoparticles (Ag NPs) are the most widely used and may be considered one of the most important. Their unique chemical and physical properties and pronounced antibacterial activity, differing largely from bulk materials, make Ag NPs a high-demand material in different sectors. Including drug delivery, medical, health care, food, consumer, industrial, household, optical and cosmetics industry [2]. Ag NPs are effective in inhibiting bacterial growth and may damage the DNA of both Gram-positive and Gram-negative bacteria [3]. Recently, Ag NPs have been frequently used in many textiles, keyboards, wound dressings, and biomedical devices. The global Ag NP market is indeed expected to reach $\$ 2.45$ billion by 2022 , with increasing demand for antimicrobial materials in healthcare applications. Healthcare is the largest sector of that market, accounting for over 30\% of the global Ag NP market revenue in 2014 [4]. With the pressing need to prevent HCAIs, it is expected that the use of Ag NPs in medical devices, equipment and textiles will further expand. Silver NPs have been used against different fungal plant pathogens due to their suppressive effects on structures and growth of fungi. There are few studies on the applicability of silver to control plant diseases [5]. Chemical reduction methods have been widely investigated for the synthesis of Ag NPs because these techniques can be executed under easy and gentle conditions, and can be used to synthesize Ag NPs on a large scale. The use of a strong reducing agent, such as sodium borohydride $\left(\mathrm{NaBH}_{4}\right)$, results in tiny particles that are well dispersed [6]. Ag NPs tend to aggregate no matter whatever routes of synthesis used and to ensure sufficient stability, stabilizing agents have to be used [7]. Such complications do not occur if the NPs are deposited on a stable inert carrier. Thus, the preparation of Ag NPs on solid supports such as kaolinite is a suitable way to prepare practically applicable supported particles as well as to control the particle size. In this work, silver will be loaded on kaolinite for stabilization and to prevent aggregation and produce silver kaolinite nanoparticless (Ag/Kln NPs). The objective of this work is to synthesis and characterize $\mathrm{Ag} / \mathrm{Kln}$ nanoparticles using synthesis technique namely chemical followed by characterization of the nanoparticles by employing X-ray diffraction (XRD), Fourier transform infrared spectra (FTIR), UV-vis spectrophotometer (UV), zeta potential measurement, scanning electron microscope (SEM) and Transmission electron microscope (TEM). The use of polymer is a prominent method for the synthesis of inorganic nanoparticles.

\section{MATERIALS AND METHOD}

\section{A. Chemical}

All reagents in this work were of analytical grade and used as received without further purification. Silver nitrate, $\mathrm{AgNO}_{3}(99 \%)$ used as the silver precursor and $\mathrm{NaBH}_{4}(99 \%)$ used as reduction agent were obtained from QRëc (New 
Zealand). Meanwhile, the kaolin powder, used as solid supporter for the Ag NPs was purchased from R \& M Chemicals (U.K). Deionized water was used in the preparation of all solutions.

\section{B. Synthesis of Ag/Kln NPs using $\mathrm{NaBH}_{4}$}

For the synthesis of $\mathrm{Ag} / \mathrm{Kln} \mathrm{NPs}$, five different concentration of $\mathrm{AgNO}_{3}$ is prepared. Constant amount of kaolin $(5 \mathrm{~g})$ were added into 5 beakers with different volume of distilled water $(250 \mathrm{~mL}, 500 \mathrm{~mL}, 1 \mathrm{~L}, 2 \mathrm{~L}, 5 \mathrm{~L})$ and stirred using a magnetic stir bar. The suspensions were then stirred for a while. Then, $0.04 \mathrm{~g}(0.5 \%), 0.06 \mathrm{~g} \mathrm{(1 \% ),} 0.16 \mathrm{~g} \mathrm{(2 \% ),}$ $0.4 \mathrm{~g}(5 \%)$ and $0.8 \mathrm{~g}(10 \%)$ of $\mathrm{AgNO}_{3}$ were simultaneously added into each respective beaker with continuous stirring and protected from light for 1 hour. Freshly prepared $\mathrm{NaBH}_{4}$ solution was added to the suspension under continuous stirring to reach a molar ratio $\mathrm{AgNO}_{3} / \mathrm{NaBH}_{4}$ (1:4). After the addition of the reducing agent, stirring was continued for another hour. The suspensions were centrifuged, filtered and washed twice using distilled water to remove the silver ion residue, and dried in an oven. All the experiments will be conducted at ambient temperature.

\section{Antibacterial activity study}

The bacterial strain Enterococcus faecalis ATCC 29212, Proteus vulgaris and Escherichia coli ATCC 25922 were obtained from the Microbiology Laboratory of Universiti Teknologi Malaysia, while Methicillin Resistant Staphylococcus aureus was obtained from Universiti Kebangsaan Malaysia. The obtained pure cultures were cultured in Mueller-Hinton agar at $37^{\circ} \mathrm{C}$ for 24 hours. They were subcultured from time to time to maintain its viability during the study period.

Mueller-Hinton broth was prepared in a Schott bottle and it was sterilised. Aliquots of the Mueller-Hinton broth were made in vials and were inoculated with the strains of $E$. faecalis, $P$. vulgaris, $E$. coli and MRSA into respective vials. The inoculated bacterial cultures were kept overnight at $37^{\circ} \mathrm{C}$.

In order to test the antibacterial activity of silver nanoparticles, Mueller-Hinton media was prepared and it was then autoclaved. Once cooled, $20 \mu \mathrm{l}$ of liquid MuellerHinton agar $\left(\mathrm{pH} 7.3 \pm 0.2\right.$ at $\left.25^{\circ} \mathrm{C}\right)$ was poured onto disposable sterile Petri dishes and allowed to solidify. Next, $6 \mathrm{~mm}$ holes were made aseptically on the solidified agar using a $6 \mathrm{~mm}$ diameter cork borer. The agar plug from each well was removed aseptically. Then $100 \mu 1$ of the microbial culture suspension in broth was spread uniformly using a disposable sterile rod and allowed to dry. Thereafter $50 \mu \mathrm{l}$ of sample and control solutions were pipetted into respective wells and allowed to diffuse completely into agar before stacking the plates upside down. The experiment was carried out in triplicate and the diameters of the zones of inhibition were measured after 24 hours of incubation at $37^{\circ} \mathrm{C}$. Commercially available antibacterial agent Gentamicin (10 $\mu \mathrm{g} / \mathrm{ml}$ ) was used as control.

\section{Statistical Analysis}

All data is presented as mean diameter of inhibition zone $(\mathrm{mm})$. The data were further analysed using a two-way ANOVA, followed by post-hoc Tukey's test using SPSS 22.0. Values of $\mathrm{p}<0.05$ indicated statistical significance among the groups tested.

\section{CHARACTERIZATION METHODS AND INSTRUMENTS}

The prepared Ag/Kln NPs were characterized by ultraviolet-visible (UV) spectroscopy, Fourier transform infrared (FTIR), zeta Potential, X-ray diffraction (XRD), scanning electron microscopy (SEM), Transmission electron microscopy (TEM). The UV-visible spectra were recorded over the 300-700 $\mathrm{nm}$ range utilizing the Shimadzu H.UV.1650 PC spectrophotometer. Transmission electron micrographs (TEM) were made in Philips CM-10 transmission electron microscope with an accelerating voltage of $100 \mathrm{kV}$. The particle size distribution was determined by using the UTHSCSA Image Tool version 2.00 program (the program was developed by Dr C.D. Wilcox and co-workers, Department of Dental Diagnostic Science, University of Texas, Health Science Center, San Antonio) [8].

The dispersion of the AgKT in the polymer matrix was evaluated at $25.0^{\circ} \mathrm{C}$ by a transmission electron microscope, TEM, (JSM-2010, JEOL) at an accelerating voltage of 100 $\mathrm{kV}$. The TEM specimen was prepared, cryotomed at low temperature $60^{\circ} \mathrm{C}$ and stained. TEM micrographs were obtained on thin cross-sections of the nanoparticles films.

FTIR spectra were recorded over the range of $500-4000$ $\mathrm{cm}^{-1}$ using Bruker Tensor II, Germany. Zeta potential was carried out using Brookhaven instrument. X-ray diffractograms were acquired using X'Pert Pro with X-ray diffractometer instrument. X-ray diffractograms were acquired using an X-ray diffractometer Pananalytical at a scanning rate of $0.324 \mathrm{~min}^{-1}$ at room temperature. The Bragg's equation was used to determine basal spacing of $\mathrm{Ag} / \mathrm{K} \ln$.

Changes in the interlamellar spacing of Kln and $\mathrm{Ag} / \mathrm{K} \ln$ NPs were studied by XRD in the angle range of $2^{\circ}<2 \theta<$ $12^{\circ}$ using $\mathrm{Cu} \mathrm{K} \alpha$ radiation $\left(\lambda=1.5406 \mathrm{~A}\right.$ ) from 10 to $80^{\circ}$. $\mathrm{X}$ ray tube voltage and current were set at $40 \mathrm{kV}$ and $40 \mathrm{~mA}$, respectively. SEM were performed using GeminiSEM 500 electron microscope to study morphology and elemental compound of $\mathrm{Kln}$ and $\mathrm{Ag} / \mathrm{K} \ln \mathrm{NPs}$. equipped with an energy dispersive spectroscopy, EDS (on X-Max 80 Oxford Instrument, Germany) using to quantify the amount of $\mathrm{Ag}$ nanoparticles. The film samples were mounted onto SEM stubs with carbon tabs attached then coated with gold using a sputter-coater.

\section{RESULTS AND DISCUSSION}

The choice of a suitable stabilizer and reducing agent is important to prepare stable Ag NPs via chemical reducing agent method. In this study, Kln suspension was used as the appropriate support for reducing $\mathrm{AgNO}_{3} / \mathrm{KLN}$ suspension using $\mathrm{NaBH}_{4}$ as the strong reducing agent. Preparation of 
silver-nanoparticles, $\mathrm{AgNO}_{3}$ solution $\left(0.01 \mathrm{~mol} \mathrm{dm}^{-3}\right)$ and kaolinite $\left(0.01 \mathrm{~mol} \mathrm{dm}^{3}\right)$ were used, respectively, as a metal salt precursor and a stabilizing agent. Upon addition of transparent colourless reaction mixture containing $\mathrm{AgNO}_{3}$ to the kaolinite suspension, the solution turns pale yellow colour. The appearance of colour indicates the formation of silver nanoparticles. As the intensities of $\mathrm{AgNO}_{3}$ increase the colour of the suspension became more intense from yellow to brown and darker in colour indicating the formation of $\mathrm{Ag}$ NPs in the kaolinite suspension. The formation of $\mathrm{Ag} / \mathrm{Kln}$ NPs was followed by measuring the surface Plasmon resonance (SPR) bands of the Ag/Kln NPs suspensions at a wavelength of 300-700 $\mathrm{nm}$. In addition, the XRD patterns in the wide angle range of $2 \theta\left(30^{\circ}<2 \theta<80^{\circ}\right)$ were also employed to determine the crystalline structures of the synthesized Ag/Kln NPs. SEM images indicated that there were no structural changes between the initial Kln and $\mathrm{Ag} / \mathrm{K} \ln$ NPs. The chemical structures of Kln and Ag/Kln NPs were analyzed using FT-IR spectroscopy.

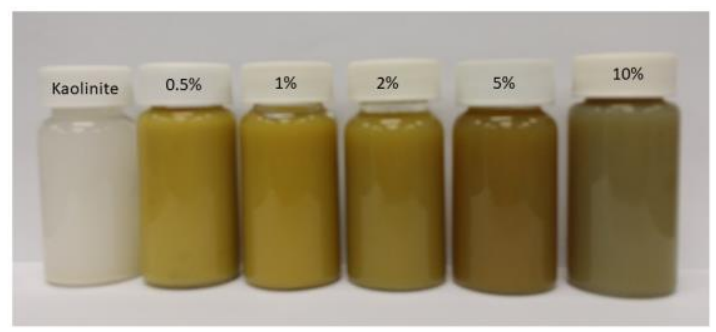

Fig.1. Photograph of Kaolin suspension (Kaolinite) and Ag/Kln NPs suspension at different $\mathrm{AgNO}_{3}$ concentrations $(0.5,1,2,5$ and $10 \%$ )

\section{A. Ultraviolet-visible spectroscopy $(U V)$}

As the aqueous solution of the silver nitrate $\left(\mathrm{AgNO}_{3}\right)$ was mixed with kaolinite suspension, it started to show the appearance of pale yellow colour due to reduction of silver ion $\left(\mathrm{Ag}^{+}\right)$, which indicated the formation of silver nanoparticles ( $\mathrm{Ag} \mathrm{NP}$ ). As the intensities of $\mathrm{AgNO}_{3}$ increase the color of the suspension became more intense from yellow to brown and darker indicating the formation of $\mathrm{Ag}$ NPs in the Kln suspension. The SPR bands are influenced by the size, shape, morphology, composition and dielectric environment of the prepared nanoparticles. The absorbance properties of various $\mathrm{Ag} / \mathrm{K} \ln \mathrm{NPs}$ suspensions prepared by sodium borohydride $\left(\mathrm{NaBH}_{4}\right)$ reduction were measured and the results are shown in Fig. 2. Notably, the characteristic of the silver SPR bands were detected in the range of 403-406 nm.

These absorption bands were presumably corresponding to Ag NPs smaller than $10 \mathrm{~nm}$. With increasing the concentration of the silver, the intensity of absorbance curves increased and they were shifted toward red wavelengths. The absorbance is directly proportional to the concentration of the nanoparticles, indicating that the absorbance of maximum absorption wavelength (kmax) increases with an increase in the number of nanoparticles. Based on this theory, nanoparticles with different sizes indicate different optical properties due to the difference in the surface plasmon resonance bands. Therefore, there was no UV-visible absorption of Ag NPs before the addition of $\mathrm{NaBH}_{4}$ (Fig. 2). Absorption peak due to SPR of Ag was slightly red-shifted to higher wavelength, it indicates the increase in the size of Ag NPs (Shameli, et al., 2010).

\section{B. Zeta Potential}

Zeta potential is an important tool for understanding the state of surface and predicting the long term stability of various colloidal dispersions of nanoparticles. The relationship between zeta potential and surface potential depends on the amount of ions in the solution. The values of the zeta potential significantly show the extent of electrostatic repulsion between all the adjacent, like charged particles in a solution or dispersion. This characterization was done using liquid sample of $\mathrm{Ag} / \mathrm{K} \ln$ NPs. Zeta potential was carried out using Brookhaven instrument. For molecules and particles in nano-scale a high zeta potential will improve the stability problems. When the potential is less this repulsion and the dispersion may break and flocculate the suspension. 


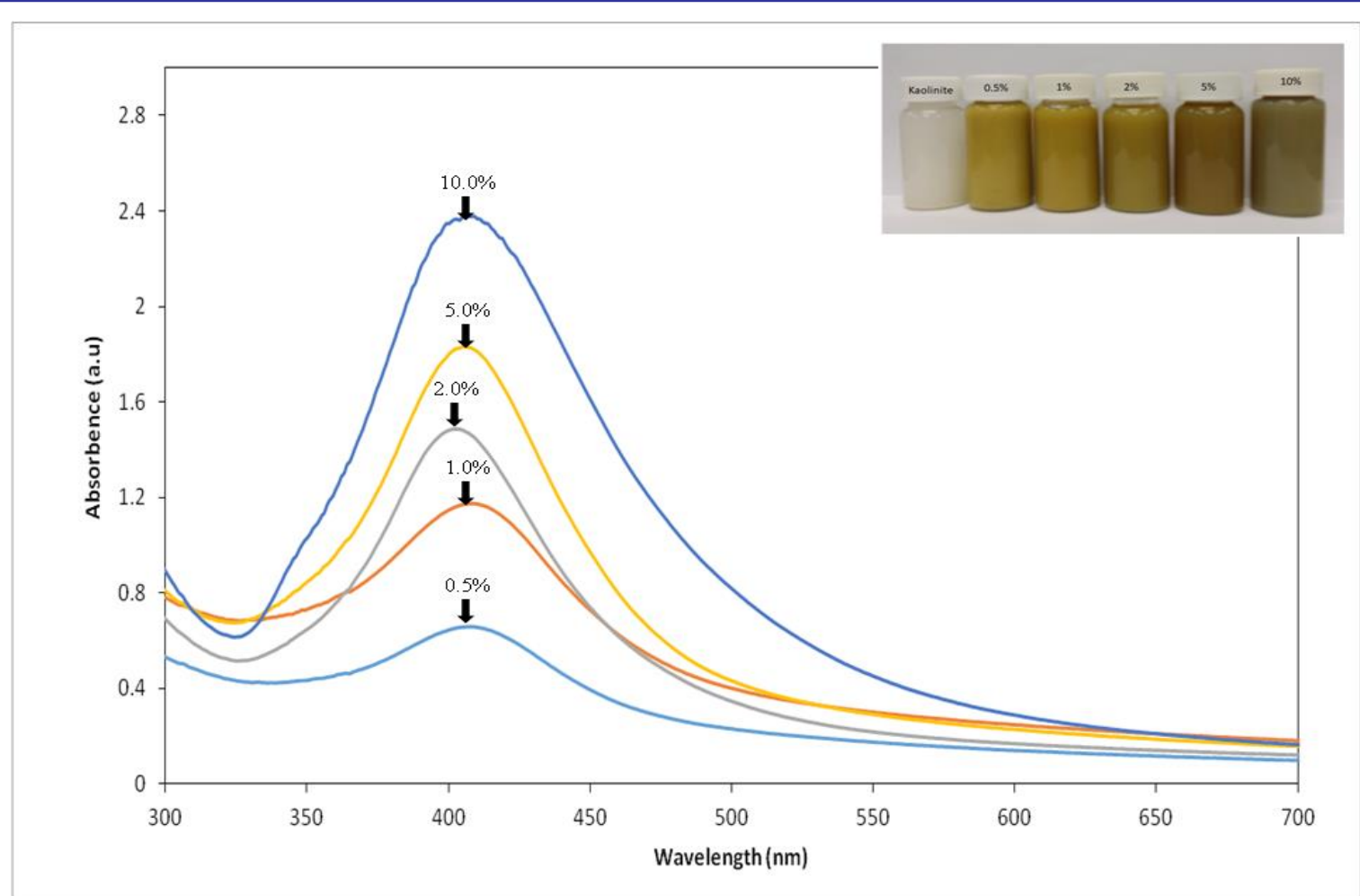

Fig. 2. UV-visible absorption spectra of $\mathrm{Ag} / \mathrm{K} \ln \mathrm{NPs}$ suspension for different $\mathrm{AgNO}_{3}$ concentration

TABLE 1. Zeta Potential at Different Concentration of Ag NP

\begin{tabular}{|c|c|c|}
\hline \multicolumn{3}{|c|}{ Zeta Potential } \\
\hline Sample & Mean $(\mathrm{mV})$ & Width $(\mathrm{mV})$ \\
\hline $0.5 \%$ & -54.12 & 2.87 \\
\hline $1.0 \%$ & -52.48 & 3.17 \\
\hline $2.0 \%$ & -52.93 & 3.08 \\
\hline $5.0 \%$ & -55.19 & 3.08 \\
\hline $10.0 \%$ & -53.13 & 3.29 \\
\hline
\end{tabular}

Suspension with high zeta potential are electrically more stabilized as compare to low zeta potentials NP (particles with zeta potentials of $\pm 30 \mathrm{mV}$ are normally considered stable). All samples showed good stability as they were between the range -52 to -55 (Table 1 ).

\section{X-ray powder diffraction (XRD)}

$X$-ray diffraction (XRD) provides information about the arrangement of atoms within a crystalline material and can detect non-bulk structures that may be observed in very small nanocomposites. The typical XRD patterns of the pure $\mathrm{Kln}$ and $\mathrm{Ag} / \mathrm{K} \ln \mathrm{NPs}$ with different concentration of $\mathrm{AgNO}_{3}$ in the small-angle range of $2 \theta\left(10^{\circ}<2 \theta<15^{\circ}\right)$, indicated that the intercalated $\mathrm{Ag}$ NPs formed between adjacent Kln lamellar layers were smaller, but the most of the Ag NPs were formed at the Kln suspension interface are presented in Fig. 3. Although the concentration of $\mathrm{AgNO}_{3}$ increased the differences in $d_{s}$ are too small and can be neglected. The original d-spacing $\left(d_{s}\right)$ of kaolinite is $0.718 \mathrm{~nm}$ at the angle of $12.31^{\circ}$. As the concentration of $\mathrm{Ag} / \mathrm{Kln}$ increases $(0.5$ $10 \%), \mathrm{d}_{\mathrm{s}}$ remained unchanged indicating there was no intercalation of silver nanoparticles in between interlayer spaces of kaolinite instead it is located on the surface of the kaolinite. Therefore, there is no increase in the basal spacing of kaolinite since all of the nanoparticles formed on the surface (Shameli et al., 2010). In addition, the XRD patterns in the wide-angle range of $2 \theta\left(30^{\circ}<2 \theta<80^{\circ}\right)$ were also employed to determine the crystalline structure of the synthesized Ag NPs (Fig. 4). Upon dispersion of Ag/Kln, four strong peaks at $2 \theta=38.11^{\circ}, 44.31^{\circ}, 64.51^{\circ}$ and $77.34^{\circ}$ correspond to the (111), (200), (220) and (311) appeared. According to the American Mineralogist Crystal Structure Database (AMCSD) [5], these peaks were attributed to the face-centred cubic silver crystals in the nanocomposite. The intensities of these sample peaks were also found to increase along with the increase in the silver nitrate concentration without any change in the position. The stronger diffraction intensity of nanocomposites is attributed to the presence of bigger crystallites or increase in crystallinity fraction due to incorporation of silver within the nanocomposite. The surface of the negatively charged layers is covered with sodium cations which can be served as the nucleating agent for different metals. 


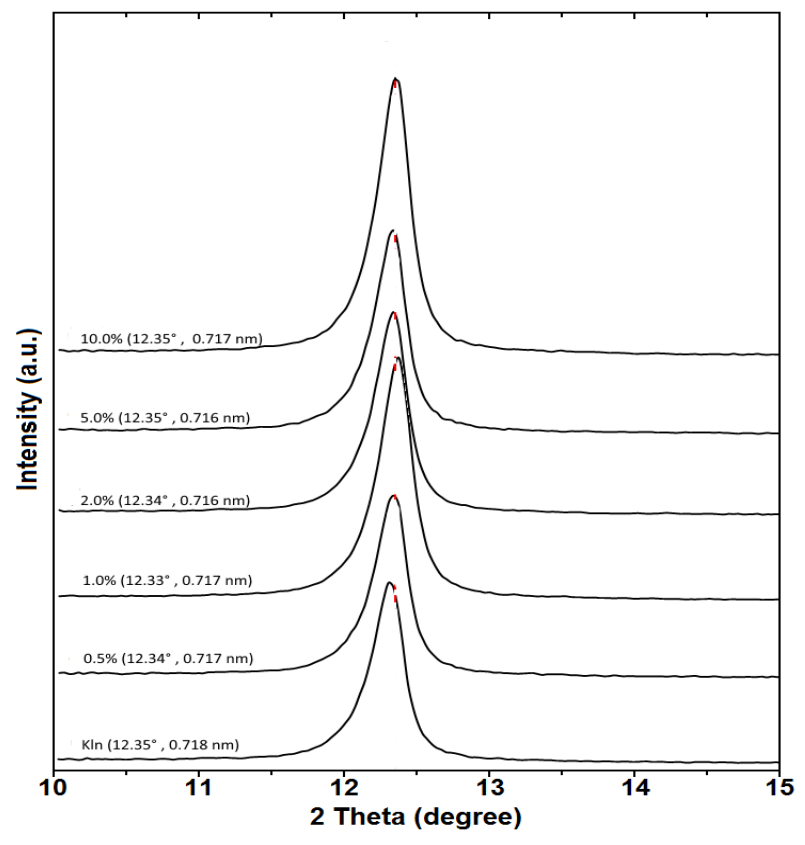

Fig. 3. XRD patterns of Kaolinite and $\mathrm{Ag} / \mathrm{Kln}$ NPs for determination of $\mathrm{d}_{\mathrm{s}}$ at different $\mathrm{AgNO}_{3}$ concentration

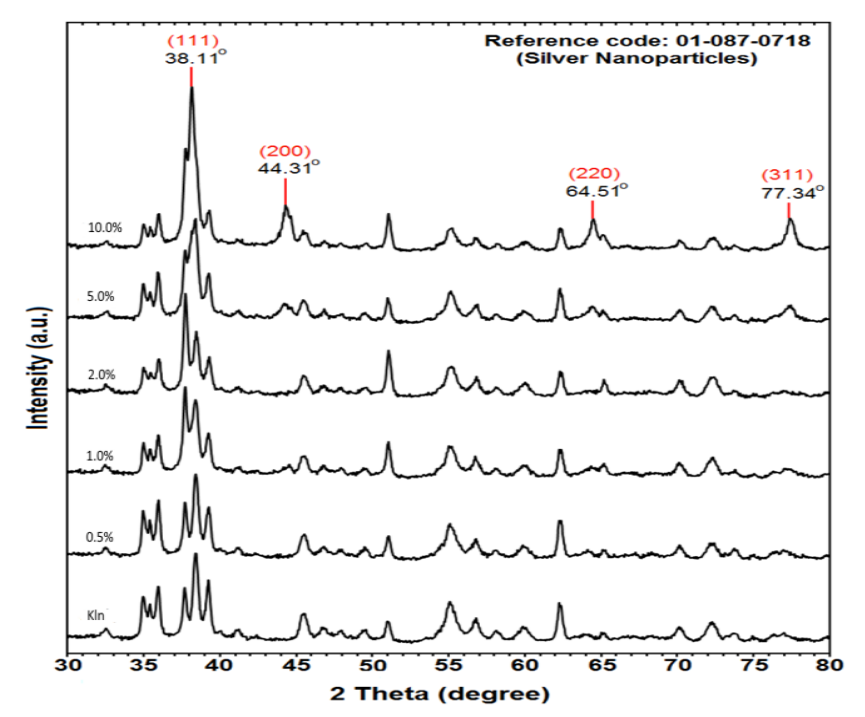

Fig. 4. XRD pattern for Ag/Kln NPs dispersion

This information includes the measurement of surface area, total pore volume, and the average pore diameter of $\mathrm{Kln}$ and $\mathrm{Ag} / \mathrm{K} \ln$ NPs.

\section{Transmission Electron Microscopy (TEM)}

TEM images and their corresponding particle size distributions of $\mathrm{Ag} / \mathrm{Kln} \mathrm{NPs}$ containing different concentration of $\mathrm{AgNO}_{3}$ are shown in Fig. 5. It is shown that all silver nanoparticles are spherical with non-uniform size. The TEM images and their size distributions revealed that the mean diameters and standard deviation of Ag NPs were about $0.56 \mathrm{~nm} \pm 0.498,0.76 \mathrm{~nm} \pm 0.976,0.88 \mathrm{~nm} \pm$ $1.173,3.67 \mathrm{~nm} \pm 6.076$ and $7.21 \mathrm{~nm} \pm 10.365$ for $0.5 \%$, $1.0 \%, 2.0 \%, 5.0 \%$ and $10.0 \%$ respectively. These results confirmed that the diameters of the Ag NPs synthesized in this method depended on the initial $\mathrm{AgNO}_{3}$ concentration.

\section{E. Scanning Electron Microscopy (SEM)}

SEM is one of the best methods to study the morphology of Kln minerals. The surface morphology of untreated Kln and $\mathrm{Ag} / \mathrm{K} \ln$ particles having different concentration are presented in Fig. 6. Kln shows surface with large flakes, which is typical structure for Kln. The silver nanoparticles loaded on surface of kaolinite were observed as regular shaped silver nanoparticles and the size of the silver nanoparticles are roughly $0.1 \mu \mathrm{m}$. Therefore, the present method can be exploited for the effective loading of Ag NPs on Kln surface. The mesoporous material of Kln has spherical shaped particles with various sizes around $1-5 \mu \mathrm{m}$.

\section{F. Chemical Analysis by FTIR}

The FTIR spectrum of parent clay mineral Kln is shown in Fig. 7. Absorptions at $3688 \mathrm{~cm}^{-1}$ in the $\mathrm{OH}$ stretching region were attributed to the inner-surface $\mathrm{OH}$ groups, while absorption at $3618 \mathrm{~cm}^{-1}$ is due to vibrations of inner hydroxyl groups (Madejová and Komadel, 2001). Very intensive bands at 1114, 1026 and $1002 \mathrm{~cm}^{-1}$ belong to $\mathrm{Si}-\mathrm{O}$ stretching vibrations. Two bands at 935 and $911 \mathrm{~cm}^{-1}$ correspond to the $\delta \mathrm{OH}$ of inner-surface and inner $\mathrm{OH}$ groups, respectively. Si-O deformation bands are located in the $787-532 \mathrm{~cm}^{-1}$ region.

As shown in Fig 7, there were not many changes in the spectra of $\mathrm{Ag} / \mathrm{Kln} \mathrm{NPs}$ compared with Kln. The FTIR spectra demonstrated the inflexibility of silicate layers and non-bond chemical interface between the silicate layers and $\mathrm{Ag}$ NPs in Ag/Kln NPs. 


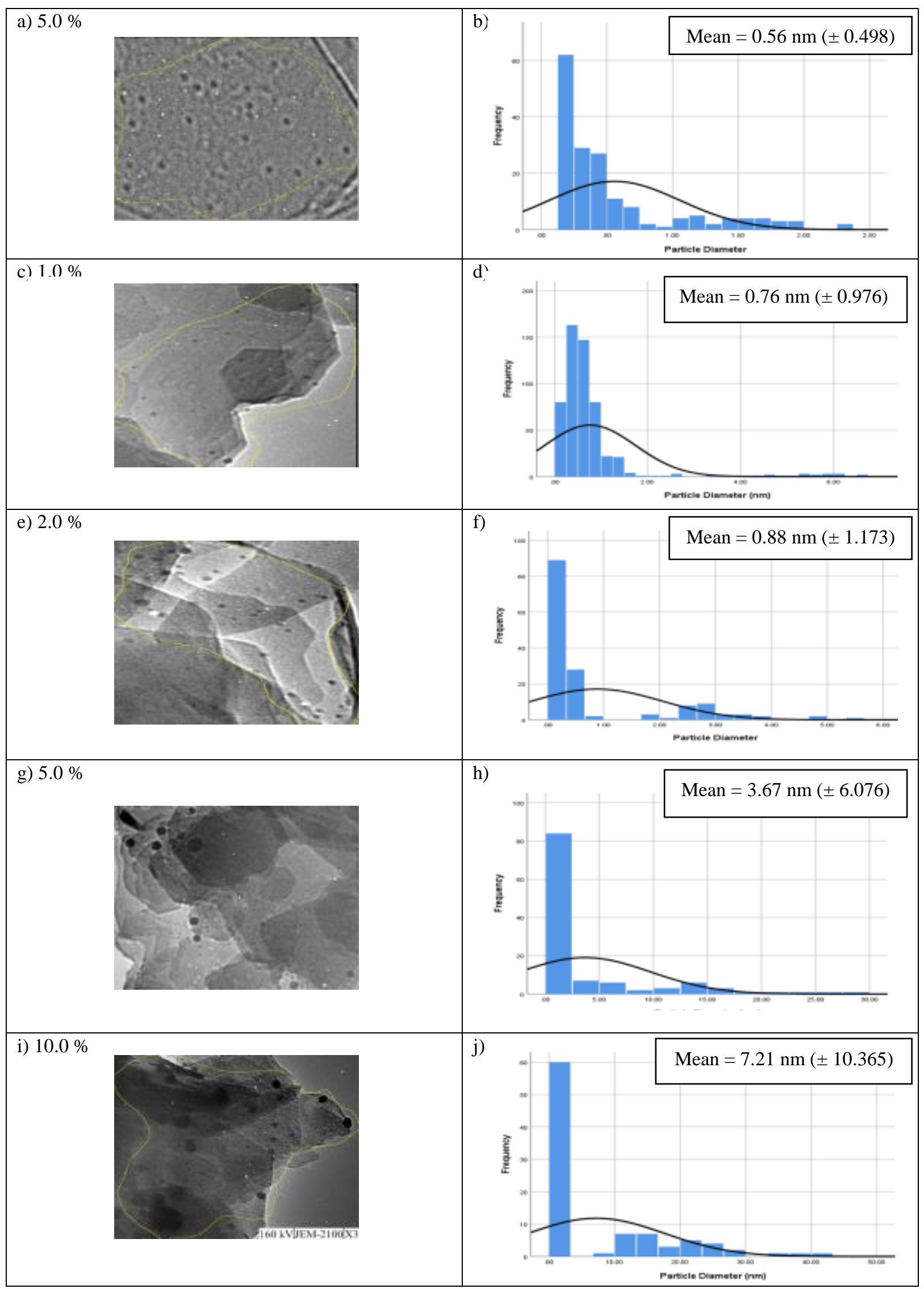

Fig. 5. TEM image of silver Nanoparticle stabilizer with kaolinite. 

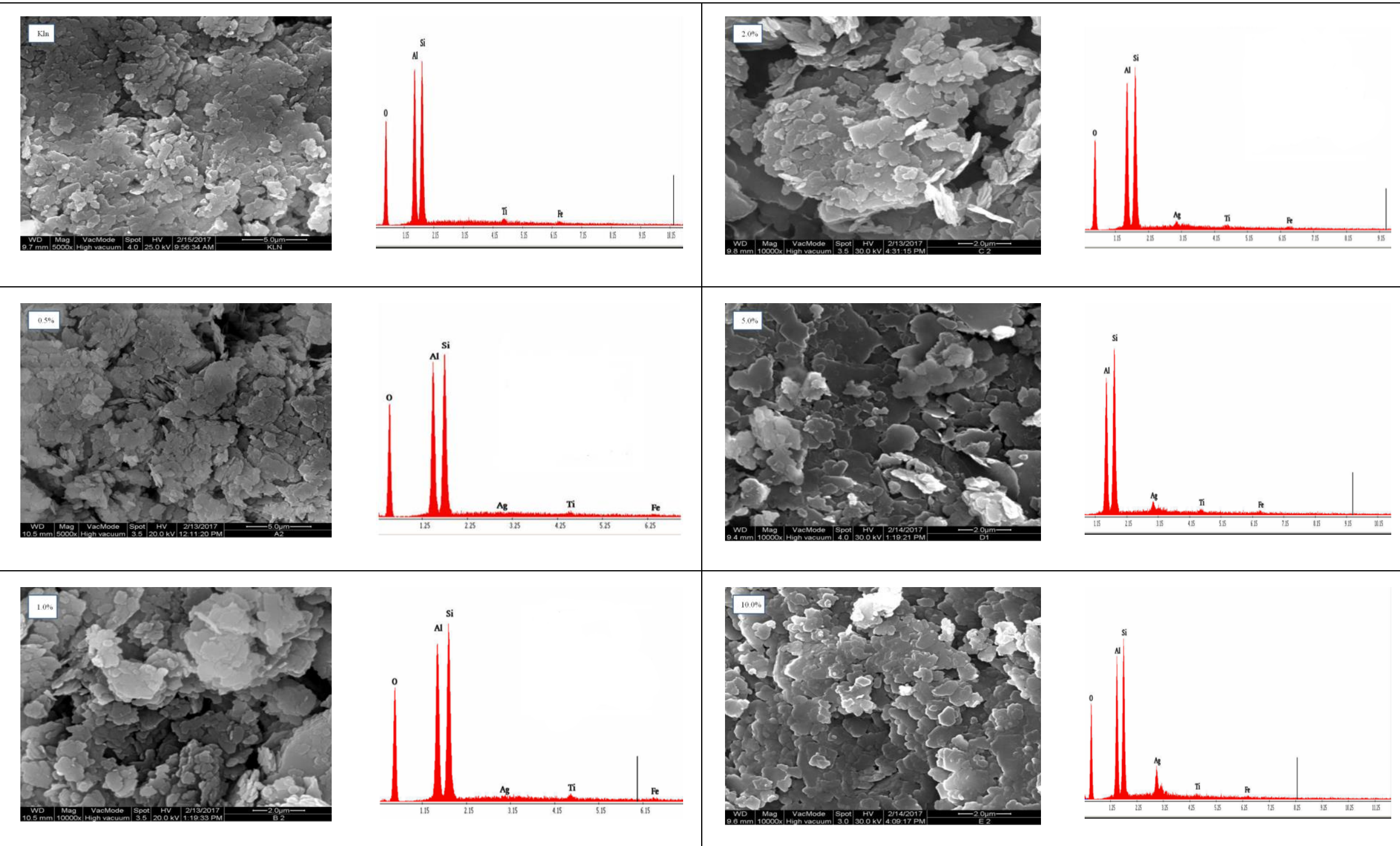

Fig. 6. SEM image of Ag/Kln NPs and EDX spectra at different concentration 


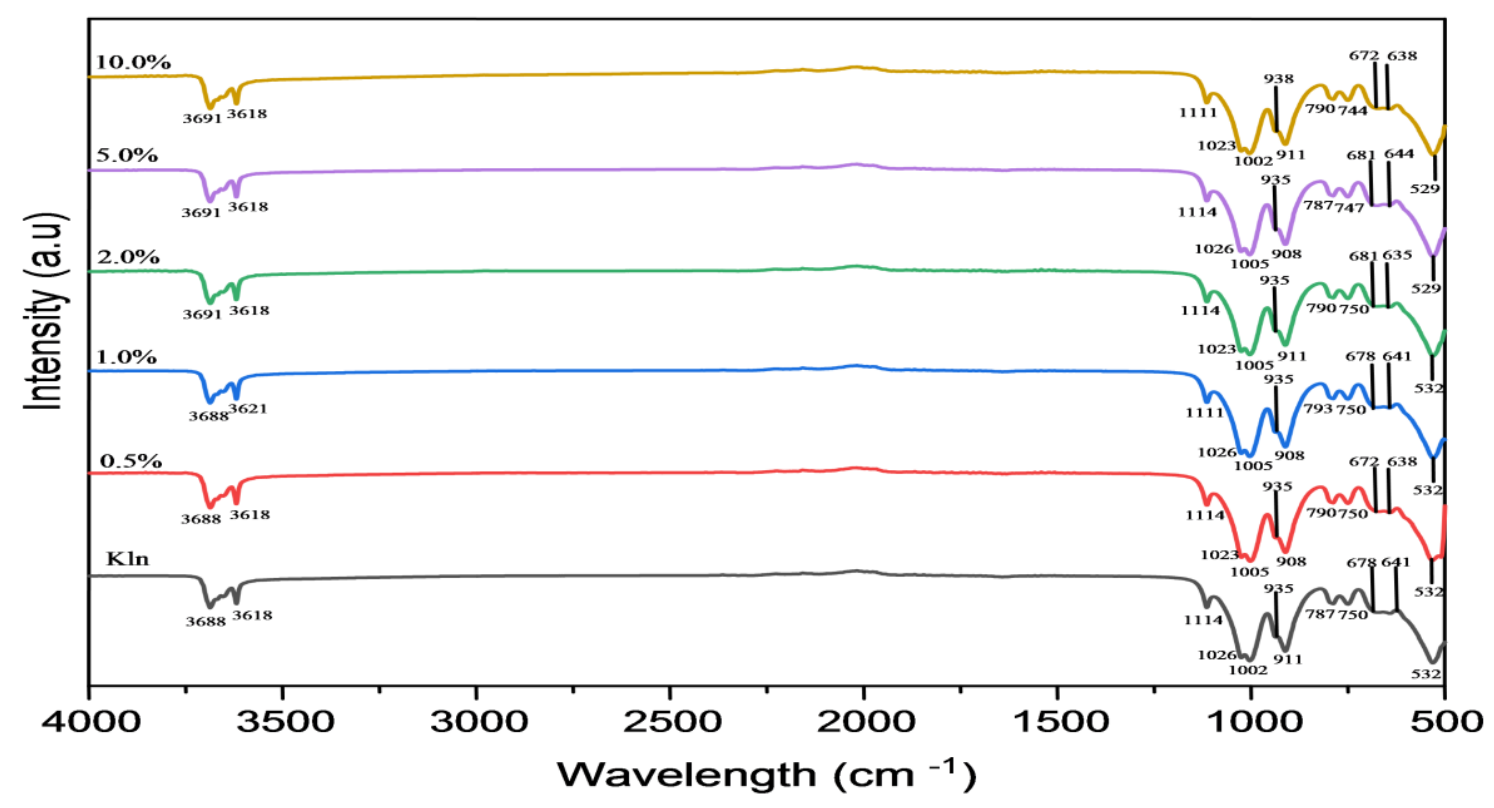

Fig. 7. FT-IR spectra for the $\mathrm{Kln}$ and $\mathrm{Ag} / \mathrm{K} \ln \mathrm{NPs}$ at different $\mathrm{AgNO}_{3}$ concentrations

\section{ANTIBACTERIAL ACTIVITY}

Inhibition zone values were obtained for the synthesized $\mathrm{AgNO}_{3} / \mathrm{K} \ln$ suspension and $\mathrm{Ag} / \mathrm{K} \ln \mathrm{NPs}(0.5 \% .1 .0 \%, 2.0 \%$, $5.0 \%$ and $10.0 \%)$ tested against E. coli, P. vulgaris, E. faecalis and MRSA. The results and images of inhibition zones are presented as the average values in Table 2 and Fig. 8 . The tests were repeated three times for each sample. In this study, all microorganisms tested are affected by the $\mathrm{AgNO}_{3}$ and $\mathrm{Ag}$ NPs in Kln suspension as shown in Table 2. This can be attributed to the size of Ag NPs which makes them easily reach the nuclear content of bacteria, and since they exhibit a large surface area the contact with bacteria is greatest [9]. In general, E. coli shows a $33-42 \%$ inhibition compared to control whereas $P$. vulgaris, E. faecalis and MRSA show 32-52\%, 30-32\% and 53-56\% inhibition respectively compared to control. From Fig. 9, Ag/Kln shows an almost consistent value in inhibition size with increasing concentrations for all microorganisms although E. coli and MRSA show higher antibacterial activity compared to the rest, similar to the finding by Xuan Hoa Vu et al. (2018). According to Usman et al. (2013), metal NPs $(\mathrm{Ag}, \mathrm{Cu}, \mathrm{CuO}, \mathrm{Au})$ exhibit a wide spectrum of antimicrobial activity against different species of microorganisms and such activity has been reported against E.coli and nonresistant strain of Gram-positive bacteria such as $S$. aureus [10].

E. coli demonstrated highest antibacterial activity followed by MRSA for all concentration Ag NPs. For $E$. coli, a Gram-negative bacterium, the greater antibacterial activity may be due to its thin cell wall thus making it more susceptible to the action of $\mathrm{Ag} / \mathrm{Kln}$ compared to Grampositive

bacteria which have a thick peptidoglycan layer in their cell walls making it more resistant towards antibacterial agent. Wang et al. suggested that the most reasonable mechanism is that bacterial cell exposure to lower concentrations of NPs causes complete disintegration of the cell surface in the form of vesicles that bind to NPs

Table 2: Mean diameter of inhibition zone observed in microorganisms against $\mathrm{Ag} / \mathrm{Kln}$ at different concentrations.

\begin{tabular}{|c|c|c|c|c|c|}
\hline \multirow{2}{*}{\multicolumn{2}{|c|}{ Treatments }} & \multicolumn{4}{|c|}{$\begin{array}{l}\text { Mean diameter of inhibition zone } \\
(\mathrm{mm})\end{array}$} \\
\hline & & \multirow{2}{*}{$\begin{array}{c}\text { E. } \\
\text { coli } \\
9.0\end{array}$} & \multirow{2}{*}{$\begin{array}{c}P . \\
\text { vulgaris } \\
7.0\end{array}$} & \multirow{2}{*}{$\begin{array}{c}E . \\
\text { faecalis } \\
6.7\end{array}$} & \multirow{2}{*}{$\begin{array}{c}\text { MRS } \\
\text { A } \\
7.3\end{array}$} \\
\hline $\mathrm{Ag} / \mathrm{Kl}$ & $0.5 \%$ & & & & \\
\hline $\mathrm{n}$ & $1.0 \%$ & 8.0 & 7.0 & 6.7 & 7.3 \\
\hline & $2.0 \%$ & 8.7 & 7.0 & 7.0 & 8.3 \\
\hline & $5.0 \%$ & 9.3 & 6.7 & 7.0 & 7.7 \\
\hline & $\begin{array}{c}10.0 \\
\%\end{array}$ & 8.3 & 7.0 & 7.0 & 7.3 \\
\hline \multicolumn{2}{|c|}{$\mathrm{Gm}(10 \mu \mathrm{g} / \mathrm{ml})$} & $\begin{array}{c}24 . \\
3\end{array}$ & 21.7 & 13.3 & 17.3 \\
\hline
\end{tabular}

Table 3: Percentage of inhibition of treatment compared to Gentamicin for $\mathrm{Ag} / \mathrm{Kln}$ at different concentrations.

\begin{tabular}{|c|c|c|c|c|c|}
\hline \multicolumn{2}{|c|}{ Treatments } & \multicolumn{4}{c|}{ \% inhibition treatment to Gm } \\
\cline { 2 - 6 } \multicolumn{2}{|c|}{} & $\begin{array}{c}E . \\
\text { coli }\end{array}$ & $\begin{array}{c}\text { P. } \\
\text { vulgaris }\end{array}$ & $\begin{array}{c}\text { E. } \\
\text { faecalis }\end{array}$ & MRSA \\
\hline \multirow{3}{*}{$\begin{array}{c}\mathrm{Ag} / \mathrm{K} \\
\ln \end{array}$} & $0.5 \%$ & 37.0 & 32.3 & 50.4 & 42.2 \\
\cline { 2 - 6 } & $1.0 \%$ & 32.9 & 32.3 & 50.4 & 42.2 \\
\cline { 2 - 6 } & $2.0 \%$ & 35.8 & 32.3 & 52.6 & 48.0 \\
\cline { 2 - 6 } & $5.0 \%$ & 38.3 & 30.9 & 52.6 & 44.5 \\
\cline { 2 - 6 } & $\begin{array}{c}10.0 \\
\%\end{array}$ & 34.1 & 32.3 & 52.6 & 42.2 \\
\hline
\end{tabular}



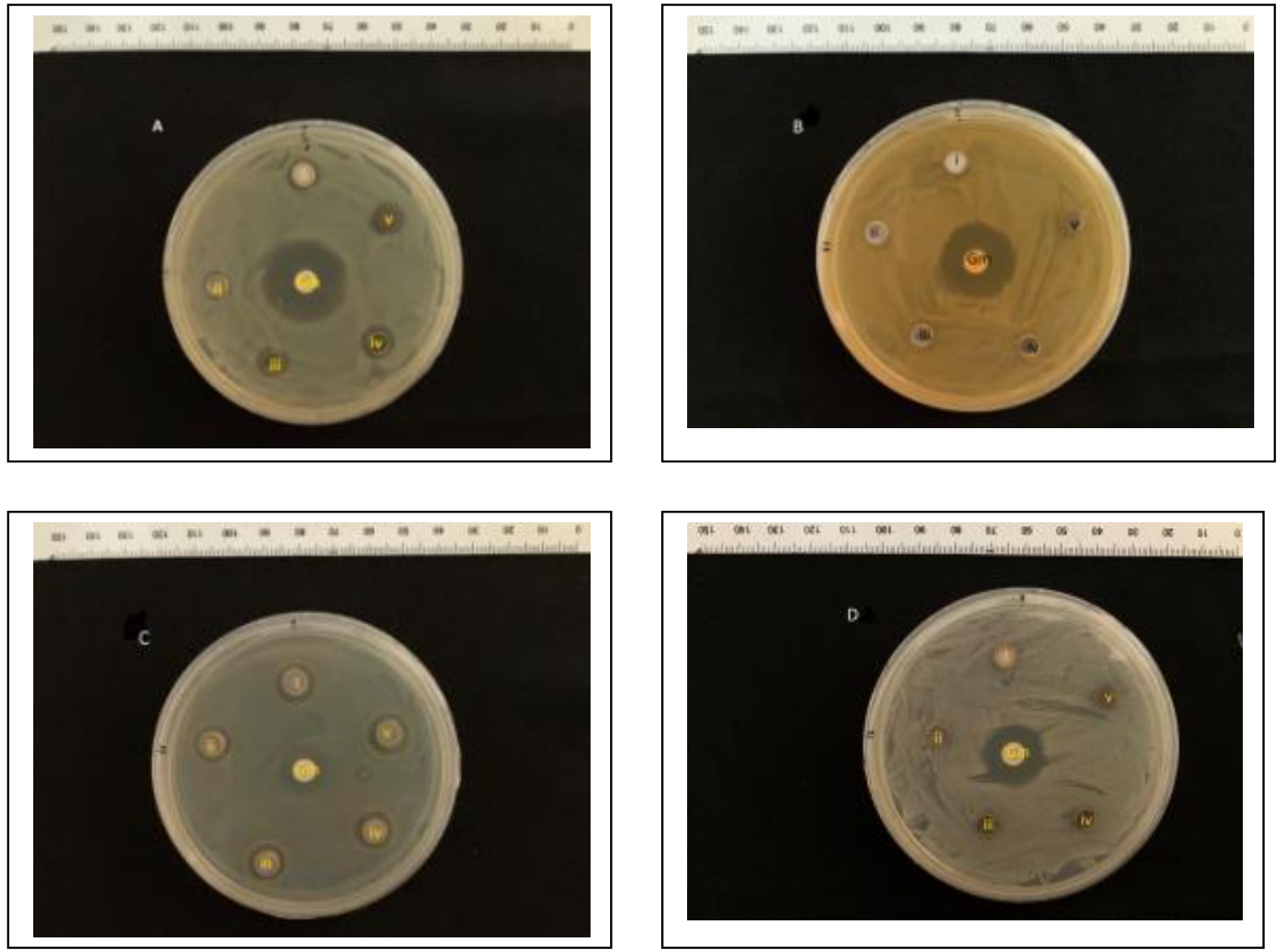

Fig. 8. (Clockwise from left) Inhibition zone of A) E.coli, B) P. vulgaris, C) E. faecalis and D) MRSA against Ag/Kln synthesised chemically at i) $0.5 \%$; ii) $1.0 \%$; iii) $2.0 \%$; iv) $5.0 \%$; and v) $10.0 \%$. Gentamicin $(\mathrm{Gm}, 10 \mu \mathrm{g} / \mathrm{ml})$ is used as positive control

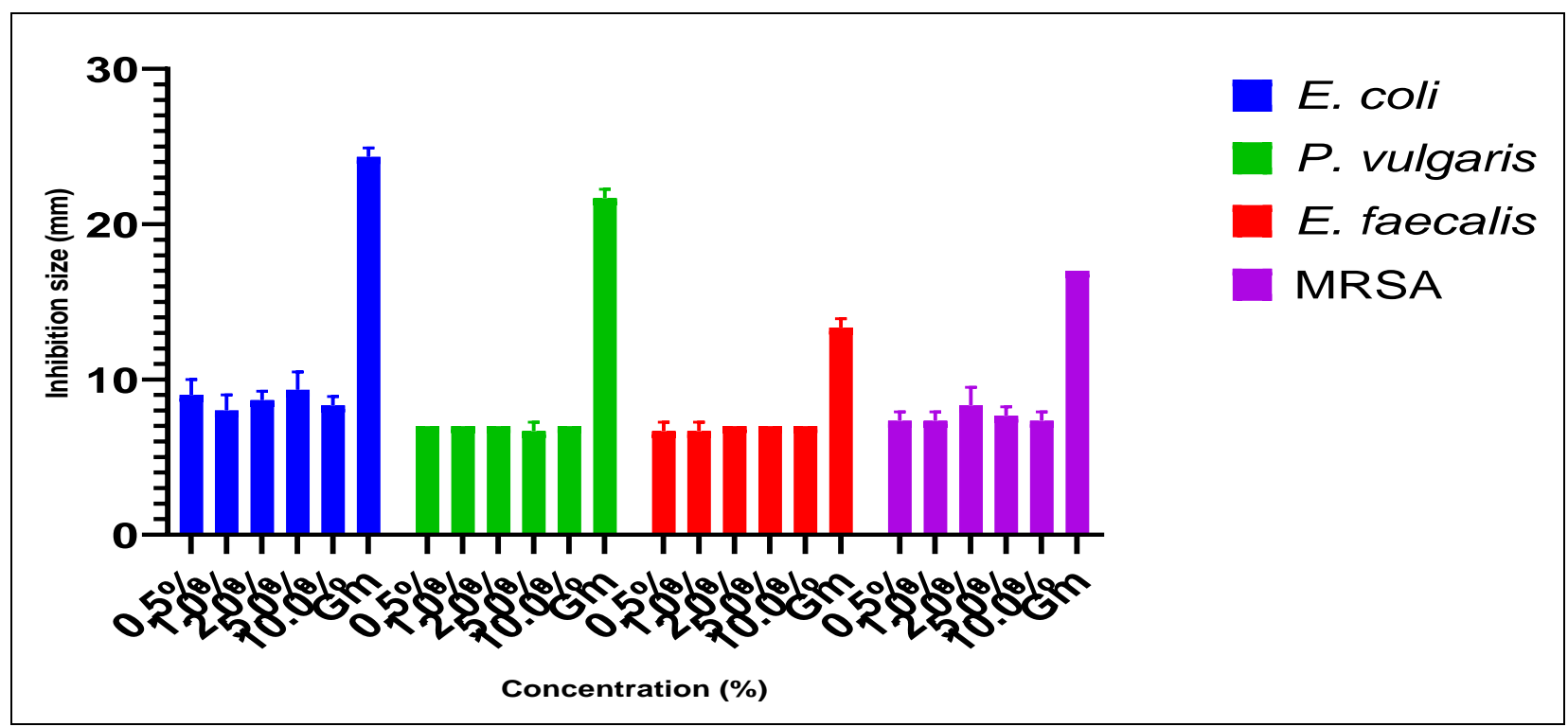

Fig. 9. Inhibition trend of microorganisms when subjected to treatments with chemically synthesised $\mathrm{Ag} / \mathrm{Kln}$.

and eventually enter the cell by electrostatic attraction [11]. There is no distinct trend observed in the antibacterial activity of Ag NPs with different concentrations although lower concentrations of Ag NP exhibits smaller particle size.
However, upon t-test analysis, a significant difference in the antibacterial efficacy of $\mathrm{Ag}$ NPs relative to control was observed for all microorganisms with $\mathrm{p} \leq 0.0001$. This indicated that the extraction method significantly influenced 
the antibacterial activity, which might also be affected by the size of the Ag NPs (Katas et al. 2018)[12].

\section{CONCLUSION}

In this study, a simple and versatile approach for the synthesis of Ag NPs has been successfully synthesized by chemical synthesis using $\mathrm{NaBH}_{4}$ from different concentrations of $\mathrm{AgNO}_{3}$. This method allows direct synthesis of Ag NPs by stirring a mixture solution consisting of $\mathrm{AgNO}_{3}$ and Kaolinite at room temperature and reduction by sodium borohydride without any other toxic and capping agents or sophisticated machines. The Ag/Kln NPs have been and Kln suspension at room temperature and are characterized by using SEM, UV, FTIR spectrometry, zeta potential, TEM and XRD techniques. The UV-visible absorption spectra showed the peak characteristic of the SPR bond of Ag NPs. The concentration of Ag salt plays a vital role in controlling the size of particles. Transmission electron microscope, TEM results concluded that the particle diameter was almost ranged between $0.56-7.21 \mathrm{~nm}$, where it increased by increasing the concentration. XRD patterns of the synthesized samples revealed that the present samples have been formed in the crystalline phase and have face center cubic lattice structure of pure silver element. Thereby, the reducing agent used in this work is suitable to produce pure Ag NPs. Therefore, the present method can be exploited for the effective loading of silver nanoparticles on Kln surface. This work also reveals a quick route to synthesize Ag NPs economically on large scale volumes using industrial grade chemicals and maintaining the purity and characteristics. The synthesized Ag-NPs exhibited substantial antibacterial activity against gram-negative bacteria, E. Coli followed by MRSA. The $2 \%$ and $5 \%$ concentrations are suggested to be the optimal concentration due to its higher antimicrobial activity against the bacteria tested. In future, more scientific researches to synthesize silver kaolinite nanoparticles using green technology will be carried out and new antimicrobial agent in combating pathogenic microorganisms for example in plant protection application can be formulated [13].

\section{REFERENCES}

[1] Gupta D, Chauhan P (2017) Green Synthesis of Silver Nanoparticles Involving Extract of Plants of Different Taxonomic Groups. J Nanomed Res 5(2): 00110. DOI: 10.15406/jnmr.2017.05.00110

[2] Zhang, Xi-Feng \& Liu, Zhi-Guo \& Shen, Wei \& Gurunathan, Sangiliyandi. (2016). Silver Nanoparticles: Synthesis, Characterization, Properties, Applications, and Therapeutic Approaches. International Journal of Molecular Sciences. 17. 1534. 10.3390/ijms17091534.

[3] Franci G, Falanga A, Galdiero S, et al. Silver nanoparticles as potential antibacterial agents. Molecules. 2015;20(5):8856-8874. Published 2015 May 18. doi:10.3390/molecules20058856

[4] Marassi V, Di Cristo L, Smith SGJ, et al. Silver nanoparticles as a medical device in healthcare settings: a five-step approach for candidate screening of coating agents. $R$ Soc Open Sci. 2018;5(1):171113. Published 2018 Jan 31. doi:10.1098/rsos.171113

[5] Ahmed AIS (2017) Chitosan and Silver Nanoparticles as Control Agents of Some Faba Bean Spot Diseases. J Plant Pathol Microbiol 8: 421. doi: 10.4172/2157-7471.1000421

[6] ma, Hongchao \& Xue, Xin-Zhong \& Liao, Jian-Hui \& Liu, ChangPeng \& Xing, Wei. (2006). Effect of borohydride as reducing agent on the structures and electrochemical properties of $\mathrm{Pt} / \mathrm{C}$ catalyst. Applied Surface Science - APPL SURF SCI. 252. 8593-8597. 10.1016/j.apsusc.2005.11.089.
[7] Calderón-Jiménez B, Johnson ME, Montoro Bustos AR, Murphy KE, Winchester MR, Vega Baudrit JR. Silver Nanoparticles: Technological Advances, Societal Impacts, and Metrological Challenges. Front Chem. 2017;5:6. Published $2017 \quad$ Feb 21 doi:10.3389/fchem.2017.00006

[8] Patakfalvi, Rita \& Oszkó, Albert \& Dekany, I. (2003). Synthesis and Characterization of Silver Nanoparticle/Kaolinite Composites. Colloids and Surfaces A: Physicochemical and Engineering Aspects. 220. 45-54. 10.1016/S0927-7757(03)00056-6.

[9] Kamyar Shameli, Mansor Bin Ahmad, Mohsen Zargar, Wan Md Zin Wan Yunus, Nor Azowa Ibrahim, Parvanesh Shabanzadeh, Mansour Ghaffari Moghaddam (2011). Synthesis and characterization of silver montmorillonite/chitosan bionanocomposites by chemical reduction method and their antibacterial activity/ International Journal of Nanomedicine (6) 271-284.

[10] Muhammad Sani Usman, Mohamed Ezzat El Zowalaty, Kamyar Shameli, Norhazlin Zainuddin, Mohamed Salama \& Nor Azowa Ibrahim (2013) Synthesis, characterization, and antimicrobial properties of copper nanoparticles. International journal of Nanomedicine (8) 4467-4479.

[11] Linlin Wang, Chen Hu \& Longquan Shao (2017). The antimicrobial activity of nanoparticles: present situation and prospects for the future. International Journal of Nanomedicine (12) 1227-1249.

[12] Ravishankar Rai, V. and Jamuna Bai, A. (2011) Nanoparticles and Their Potential Application as Antimicrobials, Science against Microbial Pathogens: Communicating Current Research and Technological Advances. In: Méndez-Vilas, A., Ed., Formatex, Microbiology Series, No. 3, Vol. 1, Spain, 197-209.

[13] Xuan Hoa Vu, Thi Thanh Tra Duong, Thi Thu Ha Pham, Dinh Kha Trinh, Xuan Huong Nguyen and Van-Son Dang (2018). Synthesis and study of silver nanoparticles for antibacterial activity against Escherichia coli and Staphylococcus aureus. Advances in Natural Sciences: Nanoscience and Nanotechnology (9) 025019 (7pp) 